\title{
ANALISIS DEL CARIOTIPO Y DETECCION DE LOS GENES 5S Y 18S/25S rDNA EN CHAETANTHERA MICROPHYLLA (CASS.) HOOK. ET ARN. (ASTERACEAE)
}

\section{KARYOTYPE ANALYSIS AND DETECTION OF 5S AND 18S/25S rDNA GENE SEQUENCES IN CHAETANTHERA MICROPHYLLA (CASS.) HOOK. ET ARN. (ASTERACEAE)}

\author{
Carlos M. Baeza ${ }^{1} \&$ Otto Schrader ${ }^{2}$ \\ ${ }^{1}$ Departamento de Botánica, Universidad de Concepción, Concepción-Chile. cbaeza@udec.cl \\ ${ }^{2}$ Federal Centre for Breeding Research on Cultivated Plants, Institute of Horticultural Crops, Neuer Weg 22/23, 06484 \\ Quedlinburg, Germany
}

\begin{abstract}
Double fluorescence in situ hybridization was used to examine the karyotype of Chaetanthera microphylla (Cass.) Hook et Arn. from Chile, including the location of 5S and $18 \mathrm{~S} / 25 \mathrm{~S}$ rDNA gene sequences. The species is $2 \mathrm{n}=24$, with $8 \mathrm{~m}+4$ st chromosomes. Signals of $5 \mathrm{~S}$ and 18/25S rDNA were seen in two of the 12 chromosome pairs: $\mathrm{n}^{\circ} 2$ and 6.
\end{abstract}

Chaetanthera Ruiz et Pav. (Asteraceae, Mutisieae) incluye hierbas anuales y perennes, nativas de Sudamérica, con alrededor de 40-42 especies, de las cuales aproximadamente 20 crecen en Chile (Cabrera 1937, Davies \& Facher 2001). Grau (1987) estudió el número de cromosomas en algunas especies chilenas de la tribu Mutisieae, las cuales incluyeron a C. glabrata (DC.) Meigen $(2 \mathrm{n}=28)$, C. linearis Poepp. $(2 \mathrm{n}=22)$ y $C$. linearis Poepp. var. albiflora Phil. $(2 \mathrm{n}=24)$. Powell et al. (1974) indican para C. tenella Less. un $\mathrm{n}=14$. C. microphylla (Cass.) Hook et Arn. es una especie endémica de Chile, distribuida principalmente en las provincias centrales de Chile, desde Aconcagua hasta Bio-bío (Cabrera 1937). Esta especie no ha sido examinada citogenéticamente.

La utilidad de los datos citológicos para estudios morfológicos y taxonómicos es bien reconocida (Stebbins 1971, Jones 1970, Stuessy 1990). La hibridización in situ por fluorescencia (FISH) ha sido ampliamente usada para estudios citotaxonómicos en diferentes grupos de plantas (Zhang \& Sang 1998, Adams et al. 2000, Weiss-Schneeweiss et al. 2003). Los marcadores citogenéticos moleculares más comunes son los genes ribosomales (5S y $18 \mathrm{~S} / 25 \mathrm{~S}$ rDNA), los cuales son abundantes y muy constantes en todas las plantas vasculares superiores (Schmidt \& Heslop-Harrison 1998).

Este trabajo documenta el complemento cromosómico de $C$. microphylla y la localización de los genes 5S y 18S/25S rDNA usando hibridizaciónin situ por fluorescencia (FISH) para el objetivo de contribuir a su caracterización citogenética.

Se estudió una población de C. microphylla colectada en Chile, VIII Región, Provincia de Nuble, Comuna de Quillón, Puente El Roble, 64 m, (3645'S$\left.72^{\circ} 25^{\prime} \mathrm{W}\right), 12-X I I-2002$, C. Baeza 4177. El material de referencia está depositado en el herbario de la Universidad de Concepción (CONC).

La preparación de los cromosomas y mediciones, como también la preparación de las sondas de ADN e hibridización in situ por fluorescencia, se realizaron siguiendo la metodología propuesta por Baeza \& Schrader (2003). Se analizaron 10 placas metafásicas y se determinó el índice de asimetría del cariotipo (AsI \%) usando la fórmula descrita por Arano y Saito (1980). Los cromosomas se clasificaron de acuerdo a Levan et al. (1964).

Chaetanthera microphylla presenta un $2 \mathrm{n}=24$ cromosomas, con un cariotipo asimétrico y un complemento cromosómico haploide de $8 \mathrm{~m}+4 \mathrm{st}$ 
(Tabla I). El cuociente entre el par más largo y el más corto $(\mathrm{R})$ fue 2,15. El índice de asimetría del cariotipo (AsI \%) fue de 62,25. Las señales de los genes rDNA $5 \mathrm{~S}$ y 18/25S se localizan en dos de los 12 pares cromosómicos. El cromosoma 2 tiene una doble señal en la porción terminal del brazo corto, la más distal corresponde a 5S rRNA y la otra señal corresponde a $18 \mathrm{~S} / 25 \mathrm{~S}$ rRNA. El cromosoma 6 presenta en la porción distal del brazo corto una señal de 5S rRNA. Los restantes cromosomas no presentan señales (Fig. 1). Ambos sitios de hibridización están ubicados sólo en los cromosomas metacéntricos. Luego de comparar los tipos de señales, se encontró que las señales 5S rDNA son más numerosas que las 18/25S rDNA, lo cual ya había sido indicado por otros autores en otros géneros de angiospermas (Hemleben \& Werts 1988, Sastri et al. 1992, Moscone et al. 1999). La intensidad de las señales de FISH entre los homólogos fue muy similar, lo que permite concluir que no existiría entre ellos diferencia en el número de copias de genes (Appels et al. 1980, WeissSchneeweiss etal. 2003). Es interesante hacer notar los diferentes niveles de ploidia que ocurren en el género Chaetanthera (Powell et al. 1974, Grau 1987); la presencia de $2 \mathrm{n}=22$ y 24 cromosomas en $C$. linearis podría indicar que la disploidia pudo ser un mecanismo citoevolutivo que produjo cambios en el número y estructura de los cromosomas dentro del género, situación ya observada en Boronia (Shan et al. 2003).

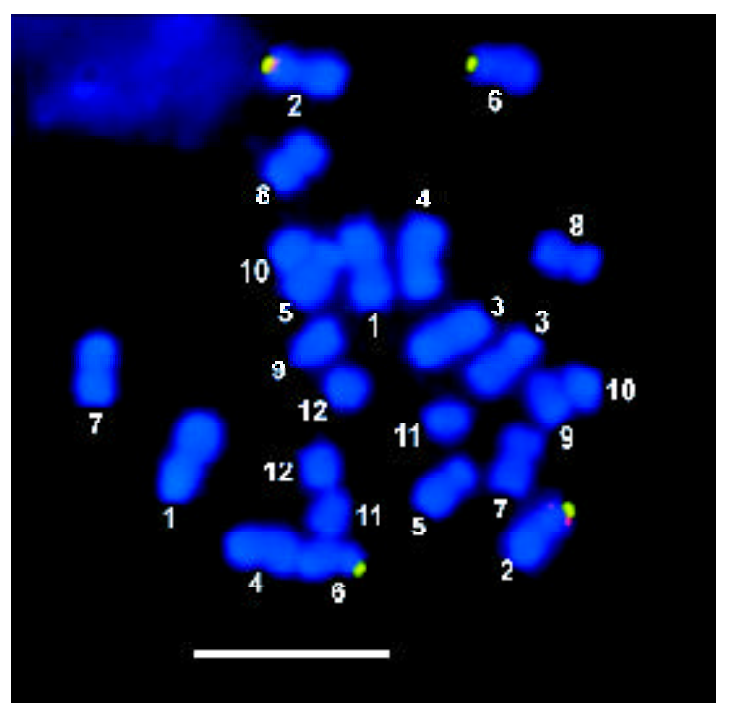

Figura 1. Cromosomas mitóticos de Chaetanthera microphylla en metafase coloreados con DAPI (azul), luego de la hibridización in situ por fluorescencia con sondas de genes rRNA específicos para 5S (amarillo) y $18 \mathrm{~S} / 25 \mathrm{~S}$ (rojo). Escala $=10 \mu \mathrm{m}$.

TABla I. Longitud promedio de los cromosomas de Chaetanthera microphylla (Cass.) Hook. et Arn., calculados en porcentaje de la longitud del genoma haploide total de 10 metafases $(93,5 \pm 9,7 \mu \mathrm{m})$. Las posiciones de las señales de fluorescencia $* 5 \mathrm{~S}$ rDNA y $* * 18 \mathrm{~S} / 25 \mathrm{~S}$ rDNA son designadas mediante asteriscos.

\begin{tabular}{|c|c|c|c|c|c|c|}
\hline $\begin{array}{c}\text { Par } \\
\text { gmosómiro }\end{array}$ & $\begin{array}{l}\text { Brofo } \\
(\%) \pm D S .\end{array}$ & $\begin{array}{l}\text { Brawo cato } \\
(\%) \pm \text { D.S. }\end{array}$ & $\begin{array}{c}\text { Larg } \\
\text { rehtiro(\%) }\end{array}$ & $\begin{array}{c}\text { Larg tothl } \\
\text { (im) }\end{array}$ & $\begin{array}{c}\text { Radiodeltrawo } \\
\text { (L/C) }\end{array}$ & $\begin{array}{c}\text { Frice } \\
\text { centromérico }\end{array}$ \\
\hline 1 & $3,02 \pm 0,12$ & $2,83 \pm 0,06$ & 5,85 & 5,47 & 107 & 0,48 \\
\hline 2 & $2,83 \pm 0,11$ & $\left.\left({ }^{*}\right) l^{*+}\right) 259 \pm 0,10$ & 5,42 & 4,86 & 1,09 & 0,48 \\
\hline 3 & $2,81+0,06$ & $2,31+0,10$ & 5,12 & 4,70 & 1,22 & 0,45 \\
\hline 4 & $2,84 \pm 0,13$ & $1,87 \pm 0,19$ & 4,71 & 4,4 & 1,52 & 0,4 \\
\hline 5 & $2,64 \pm 0,22$ & $1,80 \pm 0,29$ & 4,44 & 4,11 & 1,47 & 0,41 \\
\hline 6 & $2,53 \pm 0,10$ & $(* 1,68 \pm 0,13$ & 4,21 & 3,94 & 1,51 & 0,4 \\
\hline 7 & $2,32 \pm 0,22$ & $1,71 \pm 0,18$ & 4,03 & $3, \pi$ & 1,36 & 0,42 \\
\hline 8 & $2,09 \pm 0,15$ & $1,71 \pm 0,25$ & 38 & 3,55 & 1,22 & 0,45 \\
\hline 9 & $2,81 \pm 0,10$ & $0,67 \pm 0,02$ & 3,48 & 3,25 & 4,19 & 0,19 \\
\hline 10 & $2,61 \pm 0,14$ & $0,60 \pm 0,12$ & 3,21 & 3 & 4,35 & 0,10 \\
\hline 11 & $2,36 \pm 0,08$ & $0,56 \pm 0,12$ & 2,92 & $2, \pi 3$ & 4,21 & 0,19 \\
\hline 12 & $2,21 \pm 0,13$ & $0,51 \pm 0,06$ & $2, \pi$ & 2,54 & 4,33 & 0,19 \\
\hline
\end{tabular}




\section{AGRADECIMIENTOS}

Se agradece a la Fundación Alexander von Humboldt (Georg Foster Stipendium) y al Departamento de Botánica de la Universidad de Concepción por las facilidades otorgadas.

\section{BIBLIOGRAFIA}

Adams, S., I. Leitch, M. Bennett, M. Chase \& A. Leitch. 2000. Ribosomal DNA evolution and phylogeny in Aloe (Asphodelaceae). American Journal of Botany. 87: 1578-1583.

Appels, R., W. Gerlach, E. Dennis, H. Swift \& W. Peacock. 1980. Molecular and chromosomal organization of DNA sequences coding for the ribosomal RNAs in cereals. Chromosoma 78: 293-311.

Arano, H. \& H. Saito. 1980. Cytological studies in family Umbelliferae 5. Karyotypes of seven species in subtribe Seselinae. La Kromosomo 2: 471-480.

Baeza, C. \& O. Schrader. 2003. Karyotype analysis of Placea amoena Phil. (Amaryllidaceae) by double fluorescence in situ hybridization. Caryologia 57: 200-205.

Cabrera, A. 1937. Revisión del género Chaetanthera (Compositae). Revista del Museo de La Plata Sección Botánica 1: 87-215.

DAVIES, A. \& E. FACHER. 2001. Achene hairs and their diversity in the genus Chaetanthera Ruiz et Pav. (Mutisieae, Asteraceae). Sendtnera 7: 13-33.

Grau, J. 1987. Chromosomenzahlen chilenischer Mutisieen (Compositae). Botanische Jahrbücher 108: 229-237.

Hemleben, V. \& D. Werts. 1988. Sequence organization and putative regulatory elements in the 5S rRNA genes of two higher plants (Vigna radiata and Matthiola incana). Gene 62: 165-169.
Jones, K. 1970. Chromosome changes in plant evolution. Taxon 19: 172-179.

Levan, A, K. Fredga \& A. SAndBerg. 1964. Nomenclature for centromeric position on chromosomes. Hereditas 52: 201-220.

Moscone, E., F. Klein, M. Lambrou, J. Fuchs \& D. Schweizer. 1999. Quantitative karyotyping and dual-color FISH mapping of $5 \mathrm{~S}$ and $18 \mathrm{~S}-25 \mathrm{~S}$ rDNA probes in the cultivated Phaseolus species (Leguminosae). Genome 42: 1224-1233.

Powell, A., D. Kynos \& P. Raven. 1974. Chromosome numbers in Compositae. X. American Journal of Botany 61: 909-913.

Sastri, D., K. Hilu, R. Appels, E. Lagudah, J. Playford \& B. BAUm. 1992. An overview of evolution in plant 5S DNA. Plant Systematics and Evolution 183: 169-181.

Schmidt, T. \& J. Heslop-Harrison. 1998. Genomes, genes and junk: the large-scale organization of plant chromosomes. Trends Plant Science 3: 195-199.

Shan, F., G. Yan \& J. Plummer. 2003. Cyto-evolution of Boronia genomes revealed by fluorescent in situ hybridization with rDNA probes. Genome 46: 507513.

Stebbins, G. 1971. Chromosomal evolution in higher plants. Addison-Wesley, London, UK. 216 pp.

Stuessy, T. 1990. Plant Taxonomy. The systematics evaluation of comparative data. Columbia University Press, New York. 514 pp.

Weiss-Schneeweiss, H., T. Stuessy, S. Siljak-Yakovlev, C. Baeza \& J. Parker. 2003. Systematic and evolutionary implications of karyotypes of Hypochaeris (Asteraceae, Lactuceae) from South America. Plant Systematics and Evolution 241: 171-184.

Zhang, D. \& T. SANG. 1998. Chromosomal structural rearrangement of Paeonia brownii and $P$. californica revealed by fluorescence in situ hybridization. Genome 41: 848-853 